\title{
Feasibility of totally laparoscopic hemi- hepatectomies for liver tumor, with consideration of correspondence for difficult cases
}

\author{
Yuichiro Otsuka, Yuko Ito, Yu Matsumoto, Kazutaka Kimura, Yoji Kajiwara, Kazunori Okubo, Masaru \\ Tsuchiya, Rei Okada, Jun Ishii, Tetsuya Maeda, Kimihiko Funahashi, Hironori Kaneko \\ Division of General and Gastroenterological Surgery (Omori), Department of Surgery, Toho University Faculty of Medicine, \\ Tokyo 143-8541, Japan. \\ Correspondence to: Prof. Yuichiro Otsuka, Division of General and Gastroenterological Surgery (Omori), Department of Surgery, \\ Toho University Faculty of Medicine, 6-11-1, Omorinishi, Ota-ku, Tokyo 143-8541, Japan. E-mail: yotsuka@med.toho-u.ac \\ How to cite this article: Otsuka Y, Ito Y, Matsumoto Y, Kimura K, Kajiwara Y, Okubo K, Tsuchiya M, Okada R, Ishii J, Maeda \\ $\mathrm{T}$, Funahashi K, Kaneko H. Feasibility of totally laparoscopic hemi-hepatectomies for liver tumor, with consideration of \\ correspondence for difficult cases. Hepatoma Res 2021;7:5. http://dx.doi.org/10.20517/2394-5079.2020.112
}

Received: 24 Sep 2020 First Decision: 18 Nov 2020 Revised: 26 Nov 2020 Accepted: 14 Dec 2020 Published: 7 Jan 2021

Academic Editor: Ho-Seong Han, Luca Aldrighetti Copy Editor: Miao Zhang Production Editor: Jing Yu

\begin{abstract}
Aim: Laparoscopic liver resection (LLR) has been recognized as a minimally invasive surgery offering disease curability for liver tumors. Moreover, recent publications suggest that the systematic liver resections including hemi-hepatectomies have been performed safely in high volume centers. We describe our indication, standardized technique, and surgical outcome for totally laparoscopic hemi-hepatectomy (TLHH). Moreover, we hypothesize that TLHHs can be performed feasibly, as well as discuss the technical correspondence of technically difficult cases which have marginal indication for TLHHs.
\end{abstract}

Methods: From September 2008 to July 2020, in total, 488 cases of liver resections including 222 cases of LLR were performed in our institution. We invented the favorable indication of TLHHs of locally resectable tumors without involvement of tumor to hepatic hilus, confluence of hepatic veins, inferior vena cava, or extrahepatic organs, in patients with sufficient hepatic functional reserve for hemi-hepatectomies. Among $21 \mathrm{TLHHs}$ for liver tumors performed during study period, there were cases which derogate favorable indications; however, they might have been able to undergo TLHHs. We divided these cases into "difficult indication group (DIG)" (10 of 21 cases) and "favorable indication group (FIG)"; compared them on perioperative background, surgical outcome; and discussed the technical correspondence of TLHHs on DIG.

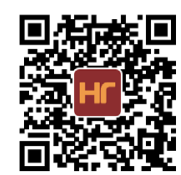


Results: There were no significant differences in patient's background and operative outcome. Operative duration, blood loss, and postoperative morbidity tended to be larger in DIG, mainly due to tumor factor, than in FIG. However, TLHHs were performed without any severe perioperative complications beyond Clavien-Dindo grade IIIb or mortality.

Conclusion: We believe that hemi-hepatectomies can safely be stylized by totally laparoscopic fashion and correspondence for difficulty can be made through technical standardization.

Keywords: Laparoscopic liver resection, major hepatectomy, laparoscopic hepatectomy, hemi-hepatectomy, liver tumor

\section{INTRODUCTION}

Laparoscopic liver resection (LLR) has been recognized as a minimally invasive surgery offering disease curability for liver tumors including hepatocellular carcinoma (HCC). Moreover, recent publications suggest that systematic liver resections including hemi-hepatectomies have been performed safely in high volume centers ${ }^{[1-5]}$. However, technical standardization of laparoscopic major hepatectomies has remained varied, depending on each institution, and has not been well established.

Surgical indication of laparoscopic approach for liver resection should be decided by patient's factors, and it should also be expanded based on the ability of each surgical unit. We introduced laparoscopic liver resection in 1993 with stringent patient selection ${ }^{[6]}$ and have applied laparoscopic major hepatectomies since 2006 by using a hybrid technique ${ }^{[7]}$. Thereafter, the totally laparoscopic approach for major hepatectomies has been performed since 2008 .

In this paper, we describe our indication, standardized technique, and surgical outcome of TLHHs. Moreover, we hypothesize that TLHHs can be performed feasibly, as well as discuss the technical correspondence of technically difficult cases which have marginal indication for TLHHs.

\section{METHODS}

From September 2008 to July 2020, in total, 222 LLRs including 21 TLHHs for liver tumors were performed at Toho University Omori Medical Center. Perioperative data from patient record were collected retrospectively, as approved by the institutional ethical committee of Toho University Omori Medical Center (ID number M19056).

\section{Surgical indications of totally laparoscopic hemi-hepatectomies}

We indicate hemi-hepatectomies for single to multiple tumors, which are limited within hemi-liver, requiring hemi-hepatectomy for curative resection.

We invented the favorable indication of TLHH is locally resectable tumor or disease without involvement into hepatic hilus, confluence of hepatic veins, inferior vena cava (IVC), or extrahepatic organs in disease factor. Tumors smaller than $7 \mathrm{~cm}$ in diameter are favorable. Sufficient hepatic functional reserve, which is tolerable for open hemi-hepatectomies calculated by Indocyanine green retention at $15 \mathrm{~min}$ (ICGR15), age, and volumetric $\mathrm{CT}^{[8]}$ are needed. Preoperative portal vein embolization performed for enlargement of remnant liver is not a contraindication for TLHH.

On the other hand, our current absolute contraindications for TLHH are tumor with significant invasion including tumor thrombus on hepatic hilus, confluence of the major hepatic veins, and IVC; requirement 


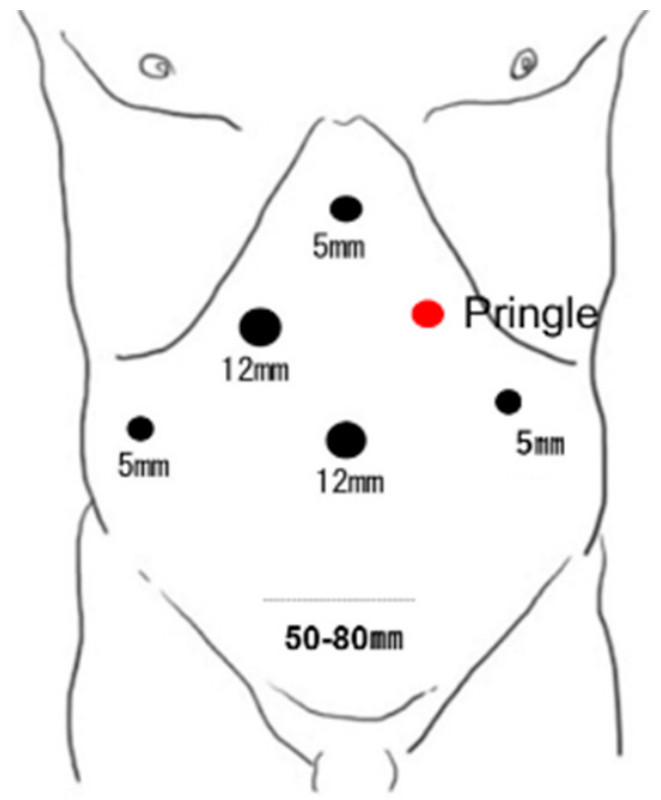

Figure 1. Trocar placement and skin incision in totally laparoscopic hemi-hepatectomies

of reconstruction of hepatic inflow, outflow vessels, or bile duct; and significant invasion to extrahepatic organs or peritoneum including dissemination. Although definite limitation is not suggested, huge tumor larger than $15 \mathrm{~cm}$ in diameter could occupy abdominal operative space even under the pneumoperitoneum. Requirement of lymphadenectomy is currently contraindicated. Patients who do not have sufficient hepatic functional reserve for hemi-hepatectomy or have ascites or coagulopathy are also contraindicated.

During our experience of 21 TLHHs, there were cases which may derogate favorable indications expected preoperatively, but they might have been able to undergo TLHH. We named these cases as "difficult indication group (DIG)" (10 of 21 cases). Difficult indication for TLHH was defined, such as for tumor with partially abutting hepatic hilus, confluence of hepatic veins, IVC, retroperitoneum, diaphragm, extrahepatic organs, or tumor larger than $7 \mathrm{~cm}$ in diameter. They were compared with "favorable indication group (FIG)" on perioperative background and surgical outcome and discussed regarding technical correspondence of TLHH on DIG.

\section{Operative procedure of pure laparoscopic hemi-hepatectomies}

Our standardized procedures for PLHH are as follows.

The patient was placed in left hemi-lateral decubitus position on right hemi-hepatectomy, and in supine position on left hemi-hepatectomy. Surgeon stood on the right side of the patient and the first assistant and scopist stood on the left side of the abdomen.

First, the laparoscope was inserted at the periumbilicus, and pneumoperitoneum was created. 4 other trocars were placed, on both sides of the abdomen, right hypochondrium, and epigastrium in both right and left hemi-hepatectomy [Figure 1].

The liver was scanned by laparoscopic flexible probe of ultrasound system through a $12-\mathrm{mm}$ trocar at right hypochondrium. Disease was confirmed by B-mode and contrast enhancement mode. Intra-hepatic structures and relation to disease were also visualized. 


\section{Hepato-duodenal ligament Extra-corporeal Rumel tourniquet}

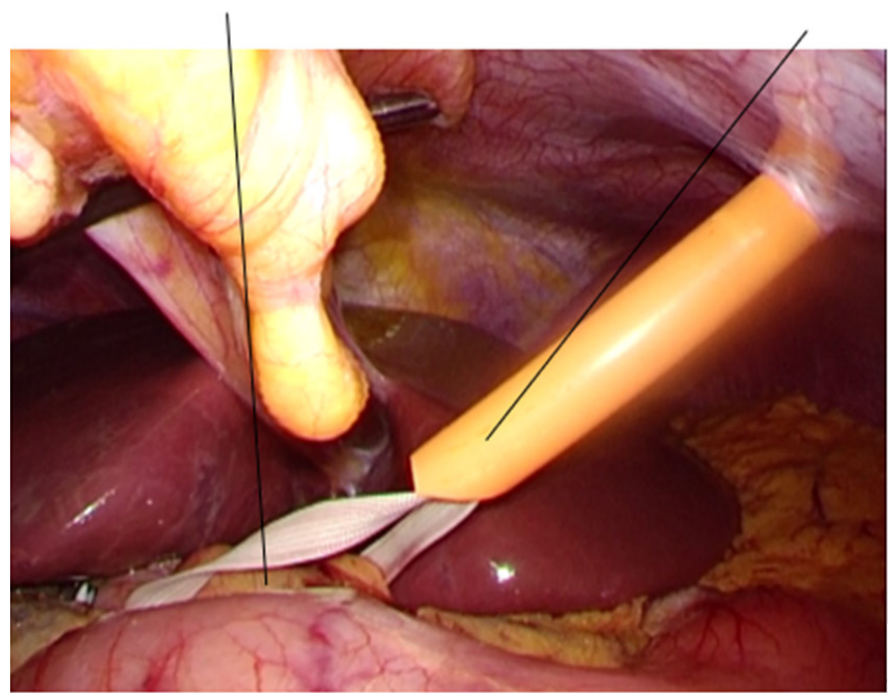

Figure 2. Extra corporeal Pringle's tourniquet

Common bile duct

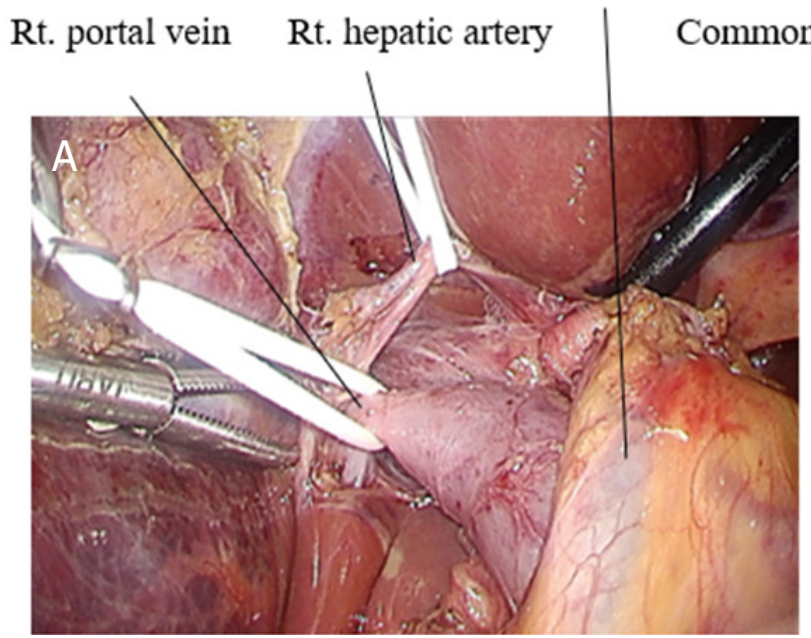

Middle hepatic artery

Lt. hepatic arteries

Figure 3. Dissection of hepatic hilum using individual isolation approach: $(A)$ hepatic hilar dissection in the right hemi-hepatectomy; and (B) hepatic hilar dissection in the left hemi-hepatectomy

After conventional laparoscopic cholecystectomy, the hepatoduodenal ligament was encircled by tape. Furthermore, an extracorporeal Rumel tourniquet for Pringle's maneuver was made using catheter on left hypochondrium [Figure 2].

For hepatic inflow control, we usually performed individual isolation technique for hepatic inflow vessels. The hepatic hilar dissection was performed. The anterior and posterior branch of the right hepatic artery in right hemi-hepatectomy and the left and the middle hepatic artery in left hemi-hepatectomy were isolated and taped. With retraction of these arteries, the right or left portal vein was dissected and isolated by tape [Figure $3 \mathrm{~A}$ and $\mathrm{B}]$.

These hepatic inflow vessels were temporarily occluded individually in order to confirm the ischemic area appeared on the liver surface. After that, the hepatic artery was doubly clipped and divided. The right or 


\section{Stapling device}

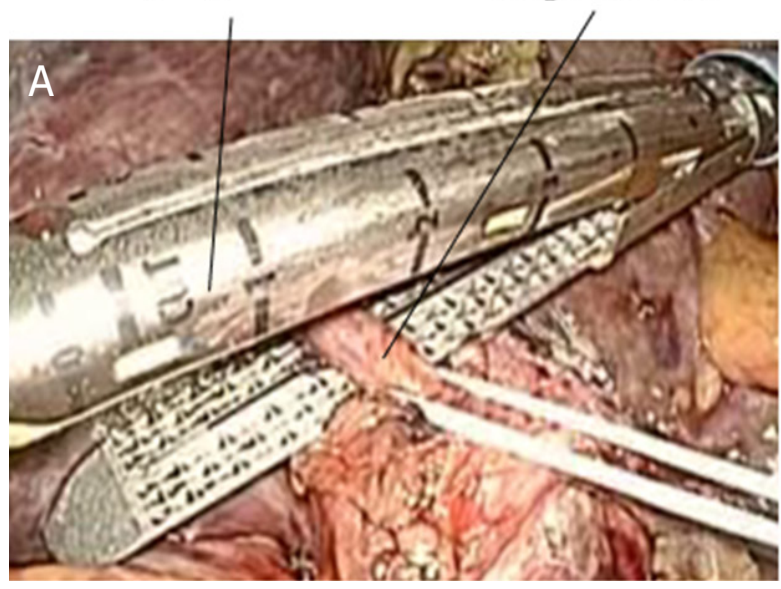

Laparoscopic scissors

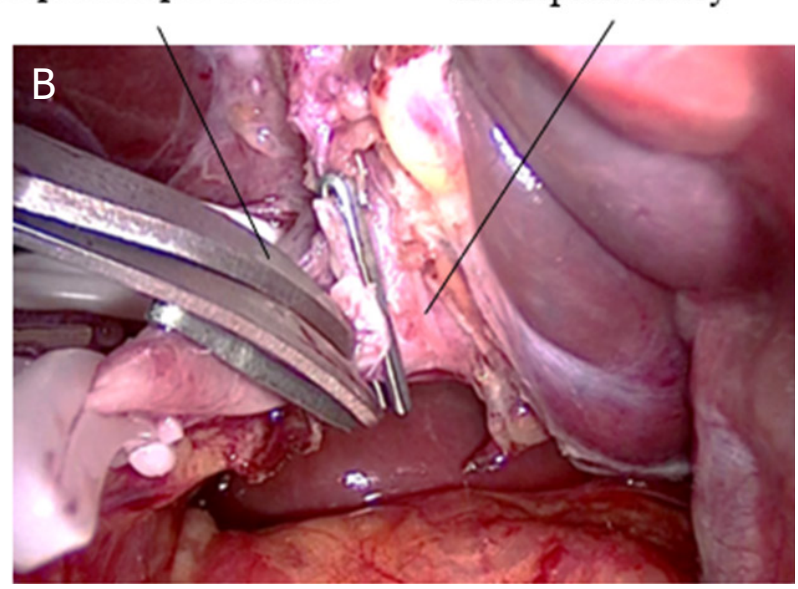

Figure 4. Division of inflow vessels: (A) division of the right portal vein using stapling device in the right hemi-hepatectomy; and (B) division of the left hepatic artery in the left hemi-hepatectomy

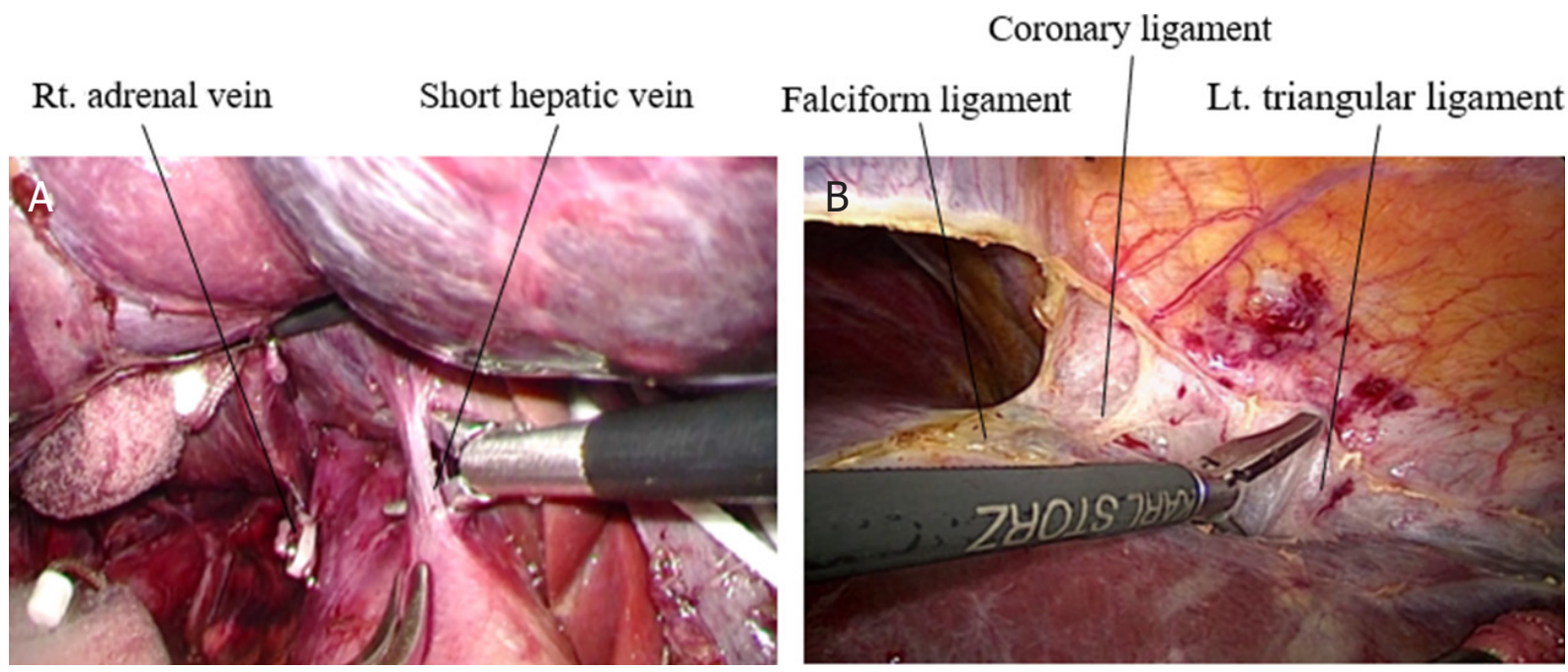

Figure 5. Mobilization of the liver: (A) mobilization of the right liver with dissection of veins from the inferior vena cava; and (B) mobilization of the left liver with dissection of surrounding ligaments

left portal vein was also doubly clipped and divided, or occasionally divided by a stapling device when the length of portal veins was long enough to insert the stapling device [Figure $4 \mathrm{~A}$ and $\mathrm{B}$ ].

Division of portal vein was occasionally performed after the liver parenchyma was divided and the hepatic hilus was opened.

Using electrocautery, a demarcation line was scored on the liver surface after division of hepatic inflow. After the inflow occlusion of the hemi-liver, the right or left liver was mobilized from the coronary ligament and the right or left triangular ligament using a bipolar sealing device.

In the right hemi-hepatectomy, the right adrenal gland was dissected from the liver and the IVC. Short hepatic veins were clipped and divided in front of the IVC. Once the root of the right or left hepatic vein was well visualized, mobilization was completed [Figure $5 \mathrm{~A}$ and $\mathrm{B}$ ]. 


\section{Cavitron ultrasonic surgical aspirator}

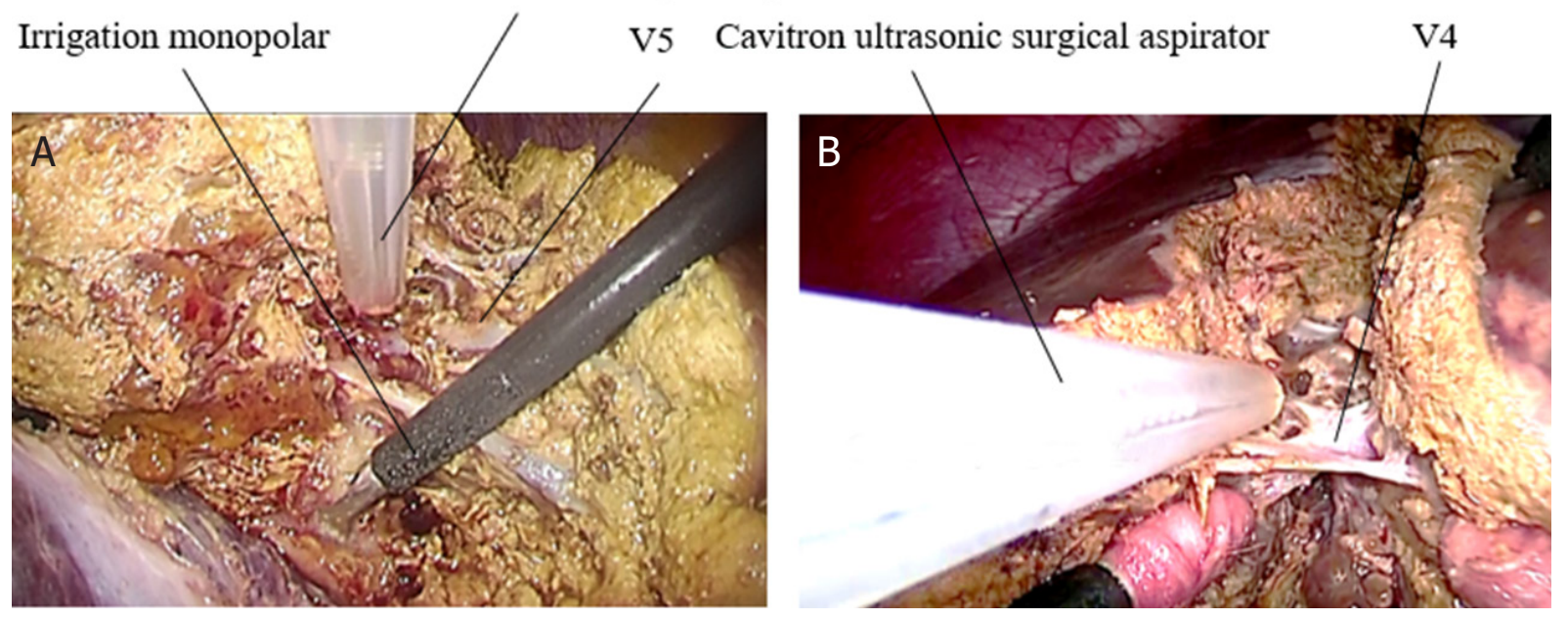

Figure 6. Transection of liver parenchyma: (A) hepatic parenchymal transection using a Cavitoron ultrasonic surgical aspirator in the right hemi-hepatectomy; and (B) hepatic parenchymal transection using a Cavitoron ultrasonic surgical aspirator in the left hemihepatectomy

Hepatic parenchymal transection was started along the demarcation line by using ultrasonic shears on superficial layer of the liver. Intra-abdominal pressure was maintained around $10 \mathrm{mmHg}$ during the entire procedure. With intermittent Pringle's maneuver, the deep liver tissue was divided using a CUSA or clamp crushing method by the bipolar sealing device. Intrahepatic vessels were meticulously exposed. Direction of parenchymal transection could usually be done from peripheral side to cranial side exposing the middle hepatic vein, with sufficient visualization of both sides of transection plane [Figure 6A and B].

Small vessels, such as Glissonean sheath less than $1 \mathrm{~mm}$ or hepatic veins less than $3 \mathrm{~mm}$, were sealed and divided by the bipolar sealing device. Large vessels were clipped and divided.

Branches of the middle hepatic vein, including veins from segments 5 and 8 in the right hemi-hepatectomy or veins from segment 4 in the left hemi-hepatectomy, were carefully isolated, clipped, and divided. The middle hepatic vein was well exposed on the resection plane.

The right hepatic duct was isolated by vessel tape, and it was divided using clips or the stapling device when the length of the hepatic duct was distally enough from the bifurcation of the right and left hepatic ducts [Figure $7 \mathrm{~A}$ and $\mathrm{B}$ ].

The confluence of the right or left hepatic vein was taped and retracted to the lateral side of the abdomen in order to insert the stapling device safely [Figure $8 \mathrm{~A}$ and $\mathrm{B}$ ].

Finally, the right or left hepatic vein was divided, and the hemi-hepatectomy was completed [Figure 9A and B].

The specimen was placed in a plastic bag along with the gallbladder and retrieved from the abdominal cavity through a suprapubic incision, usually $5-8 \mathrm{~cm}$ in length. Once the hemostasis of the resection plane was achieved, two closed suction drains were placed into the Winslow's fossa and subphrenic space. Then, the abdomen was closed.

\section{Postoperative care and discharge}

After the extubation and awaking from anesthesia, patients were transferred from the operating room to the high care unit. Ambulation and oral intake were usually started on Postoperative Day 1. Abdominal 

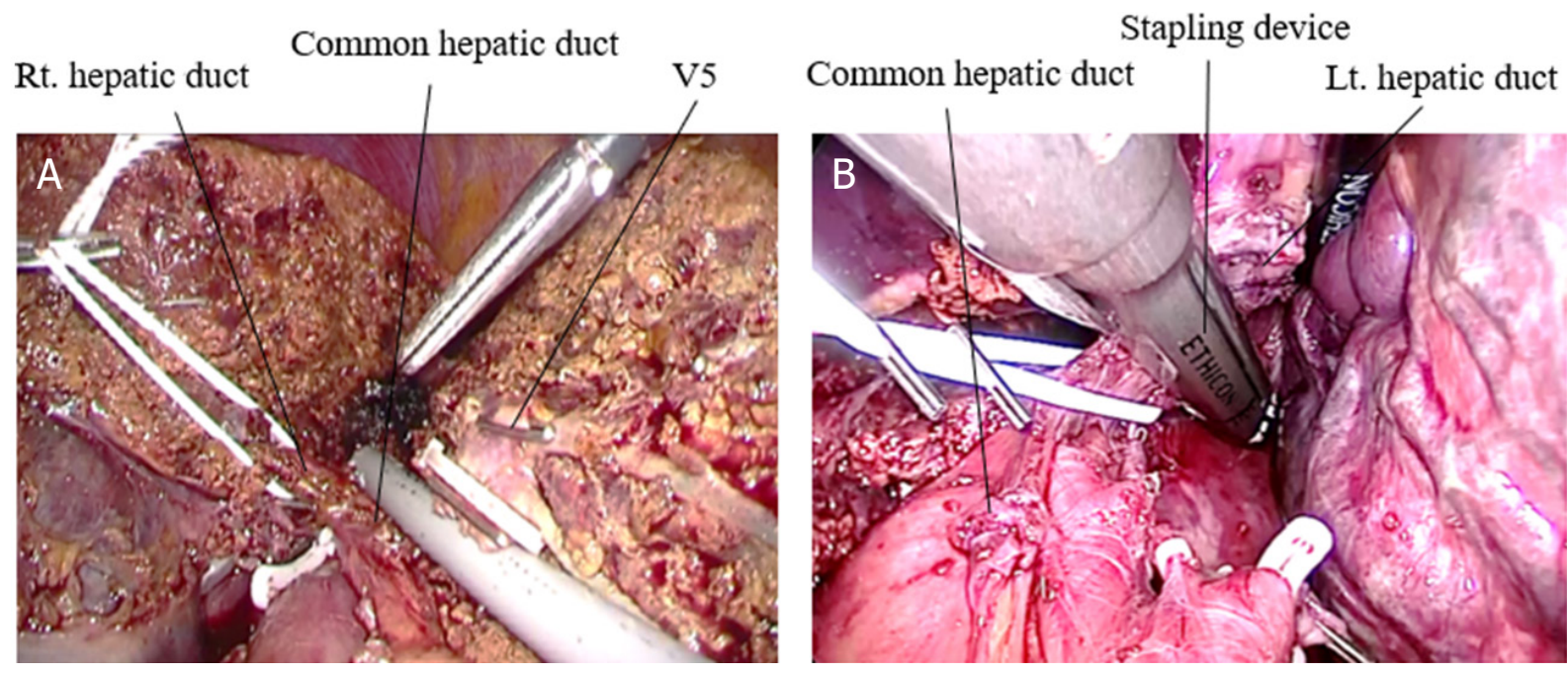

Figure 7. Isolation and division of the hepatic duct: $(A)$ isolation of the right hepatic duct in the right hemi-hepatectomy; and (B) division of the left hepatic duct using stapling device in the left hemi-hepatectomy
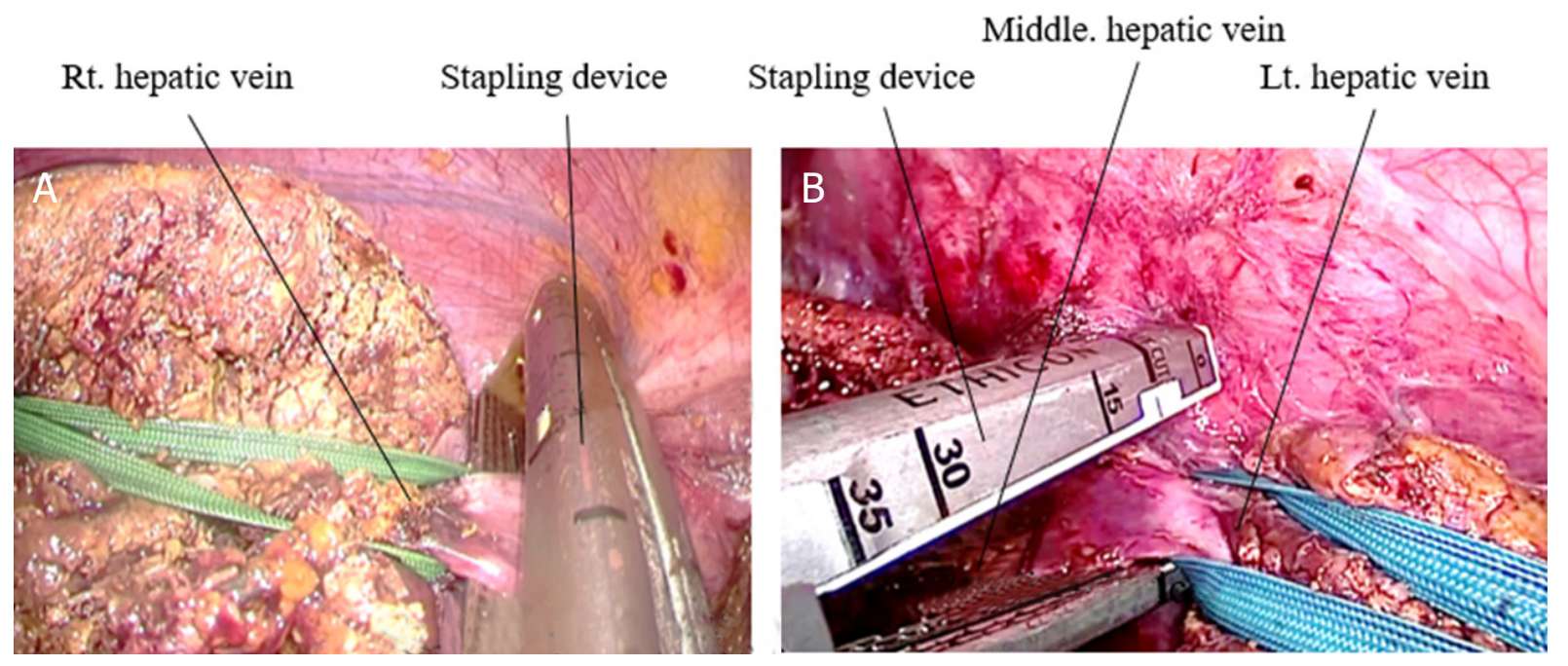

Figure 8. Division of the hepatic veins: (A) division of the right hepatic vein using stapling device; and (B) division of the left hepatic vein using stapling device

drains were removed at less than $100 \mathrm{cc}$ in volume without sign of bile leakage, hemorrhage, or infection. Patient discharge date was decided with sufficient recovery, termination of treatment for morbidity, patient activity of daily life as almost compatible with preoperative condition, and consideration of patient's family convenience.

\section{Statistical analyses}

Perioperative data of patients who received PLHH were retrospectively collected. Comparison of patient background and operative outcome between FIG and DIG was performed. Clinical parameters such as age, gender, body mass index (BMI), presence of chronic liver disease, Child-Pugh score, ICGR15, etiology of liver tumors, tumor number, tumor diameter, type of resection, operative duration, estimated blood loss, transfusion requirement, postoperative morbidity, postoperative hospital stay, and postoperative mortality were compared between the two groups. The data are expressed as median (range). Comparison of quantitative variables was performed using the Fisher exact test. The Mann-Whitney U test was used to 

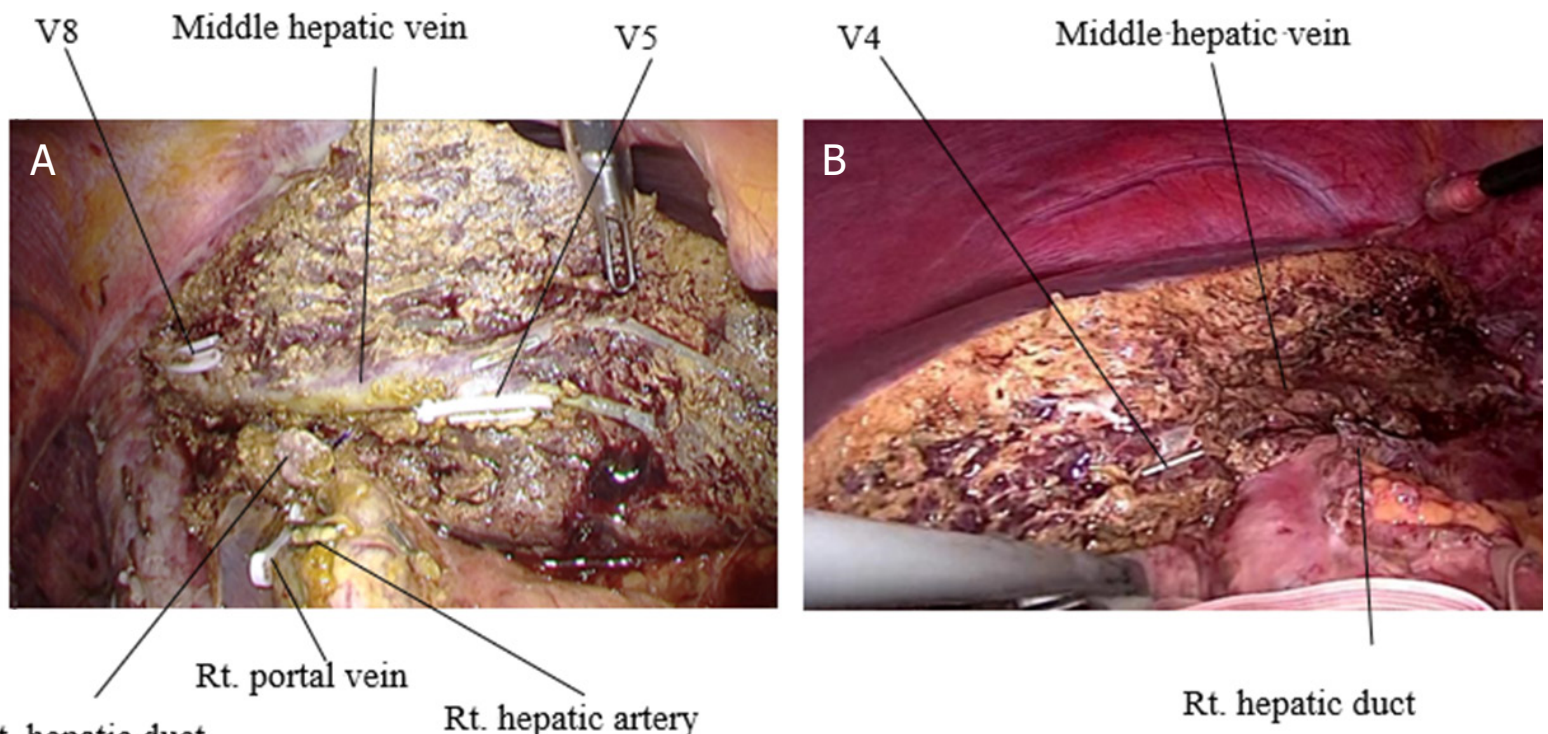

Rt hepatic duct

Rt. hepatic artery

Rt. hepatic duct

Figure 9. Resection planes of totally laparoscopic hemi-hepatectomies: (A) completion of the right hemi-hepatectomy; and (B) completion of the left hemi-hepatectomy

Table 1. Procedure and disease profiles of the performed pure laparoscopic hemi-hepatectomies

\begin{tabular}{lccc}
\hline & Rt. hemi-hepatectomy & Lt.hemi-hepatectomy & Sum \\
\hline Hepatocellular carcinoma & $6(66.7 \%)$ & $3(33.3 \%)$ & 9 \\
Intrahepatic cholangiocarcinoma & $2(66.7 \%)$ & $1(33.3 \%)$ & 3 \\
Metastatic cancer & $3(75.0 \%)$ & $1(25.0 \%)$ & 4 \\
Benign to low grade malignant tumors & $2(40.0 \%)$ & $3(60.0 \%)$ & 5 \\
Sum & $13(61.9 \%)$ & $8(38.1 \%)$ & 21 \\
\hline
\end{tabular}

compare continuous variables. Statistical analysis was performed using EZR software version $1.51^{[9]}$. A $P$ value less than 0.05 was considered statistically significant.

\section{RESULTS}

During this study period, 488 liver resections were performed in our institution, including 222 cases of laparoscopic liver resection and 266 cases of open liver resection. In laparoscopic liver resections, 94 cases were anatomical hepatectomies and 128 cases were non-anatomical partial hepatectomies. In open liver resections, 195 cases were anatomical hepatectomies and 71 cases were non-anatomical partial hepatectomies. In laparoscopic anatomical liver resections, 31 cases were major liver resections, which remove more than three segments defined by Couinaud's classification, including 25 TLHHs. In 25 TLHHs, 21 cases were performed for liver tumors, while four cases were for hepatolithiasis. In open anatomical hepatectomies, 73 cases were major hepatectomies including 69 hemi-hepatectomies.

The 21 laparoscopic hemi-hepatectomies comprise 13 right hemi-hepatectomies and 8 left hemihepatectomies. They were performed for primary or metastatic liver disease, including nine for HCC, four for colorectal cancer metastasis, three for intrahepatic cholangiocarcinoma (ICC), and five for benign to low grade malignancy liver tumors [Table 1].

Among these cases, 10 patients were considered as DIG, of which four cases had a tumor larger than $70 \mathrm{~mm}$ (80-120 $\mathrm{mm}$ ), three patients had tumor located adjacent to the confluences of major hepatic veins, one had tumor invading the right adrenal gland, one suspicion of tumor invading to the right diaphragm, and one suspicion of tumor invading the retroperitoneum. Another 11 patients were classified as FIG. 
Table 2. Patient characteristics of those who received totally laparoscopic hemi-hepatectomies

\begin{tabular}{llll}
\hline & Favorable cases $(\boldsymbol{n}=\mathbf{1 1})$ & Difficult cases $(\boldsymbol{n}=\mathbf{1 0})$ & $\boldsymbol{P}$-value \\
\hline Age & $60(41-77)$ & $53(26-79)$ & 0.382 \\
Gender (Male: Female) & $4: 6$ & $9: 2$ & 0.361 \\
Body Mass Index & $21.3(19.0-26.2)$ & $23.5(16.8-35.1)$ & 0.494 \\
Chronic liver disease & $4(36.4 \%)$ & $5(50.0 \%)$ & 1.0 \\
Child-Pugh score & $5(5-8)$ & $5(5-5)$ & 0.391 \\
ICGR $_{15}$ & $9.0(2-17)$ & $5.5(1-10)$ & 0.0576 \\
Hepatocellular carcinoma & $4(36.4 \%)$ & $5(50.0 \%)$ & 1.0 \\
Cholangiocellular carcinoma & $2(18.2 \%)$ & $1(10.0 \%)$ & 0.586 \\
Metastatic liver cancer & $1(9.1 \%)$ & $3(30.0 \%)$ & 0.586 \\
Benign to low grade malignant tumor & $2(18.2 \%)$ & $3(30.0 \%)$ & 0.635 \\
Tumor-number & $1(1-4)$ & $1(1-5)$ & 0.895 \\
Tumor-size(mm) & $47(30-55)$ & $70(33-120)$ & 0.0559 \\
Procedure (Rt.: Lt.) & $7: 3$ & $3: 5$ & 0.659 \\
\hline
\end{tabular}

$I \mathrm{CGR}_{15}$ : Indocyanine green retention at $15 \mathrm{~min}$

In the comparison of backgrounds of patients, there was no significant difference between FIG and DIG patients' age, gender distribution, BMI, presence of chronic liver disease, Child-Pugh score, and ICGR15. PLHH procedure, tumor-number, tumor-size, and distribution of tumor etiology, such as HCC, ICC, metastatic liver cancer, and benign to low grade malignant tumors, were not significantly different between the 2 groups [Table 2].

In the comparison of operative outcome, there was no significant deference in operative duration, estimated blood loss, requirement of transfusion, conversion to open surgery, postoperative morbidity, postoperative hospitalization, and postoperative mortality. Median value of operative durations in FIG and DIG were 586.0 (355-749) min and 625.5 (240-768) min, respectively. Estimated blood loss in FIG and DIG were 290.0 (10-1060) cc and 357.5 (50-2683) cc, respectively. One conversion to hybrid technique with small laparotomy was observed in a case of a right hemi-hepatectomy in DIG, due to hemorrhage from dense adhesion on the retroperitoneum, which was suspected but negative for tumor invasion. Postoperative morbidity was found in two (18.2\%) in FIG, while organ/space surgical site infection (SSI) in one and bile leakage in one. In DIG, three patients (30\%) experienced postoperative morbidity, including superficial SSI in one, bile leakage in one, and thrombus in portal vein in one. These complications were all treated by conservative treatment without surgical interventions, and no postoperative complication beyond ClavienDindo grade IIIb was found. Postoperative hospitalization was 9 (6-25) days in FIG and 13 (7-45) days in DIG. No mortality was found in either group [Table 3].

Regarding the correspondence to difficulty in DIG, we describe technical details of the issues.

For the tumor larger than $7 \mathrm{~cm}$, mobilization of hemi-liver was not possible prior to liver parenchymal transection. For this issue, obtaining operative abdominal space and visualization of hepatic hilum was important. In all four cases of large tumor, hepatic hilar dissection was successful to isolate hepatic inflow vessels. After the demarcation line appeared on the liver surface by inflow occlusion, liver transection was performed. As the transection deepened, hepatic hilum could be widely opened. Inflow vessels and bile duct were divided using clips or stapling device. After the completion of liver transection and division of hepatic veins, mobilization of removed hemi-liver was accomplished [Figure 10A-D].

For cases of tumor located adjacent to the confluences of major hepatic veins, full mobilization of hemi-liver was the most important first step. Especially, liver mobilization should be achieved by devascularization of the half of removal side on the anterior wall of the IVC with division of short hepatic veins. For the approach to the confluences of major hepatic veins, an additional trocar was placed at the 
Table 3. Comparison of operative outcome of pure laparoscopic hemi-hepatectomy

\begin{tabular}{lllc}
\hline & Favorable cases $(\boldsymbol{n}=\mathbf{1 1})$ & Difficult cases $(\boldsymbol{n}=\mathbf{1 0})$ & $\boldsymbol{P}$-value \\
\hline Operative duration & $586.0(355-749)$ & $625.5(240-768)$ & 0.251 \\
Estimated blood loss & $290.0(10-1060)$ & $357.5(50-2683)$ & 0.342 \\
Transfusion requirement & $1(9.1 \%)$ & $2(20.0 \%)$ & 0.586 \\
Postoperative morbidity & $2(18.2 \%)$ & $3(30.0 \%)$ & 0.635 \\
& SSI (organ/space) 1 (9.1\%) & SSI (superficial) 1(10.0\%) & \\
& Bile leakage 1 (9.1\%) & Bile leakage 1(10.0\%) & \\
Postoperative hospitalization & & Portal vein thrombus 1(10.0\%) & 0.111 \\
Mortality & $9(6-25)$ & $13(7-45)$ & 1.0 \\
\hline
\end{tabular}
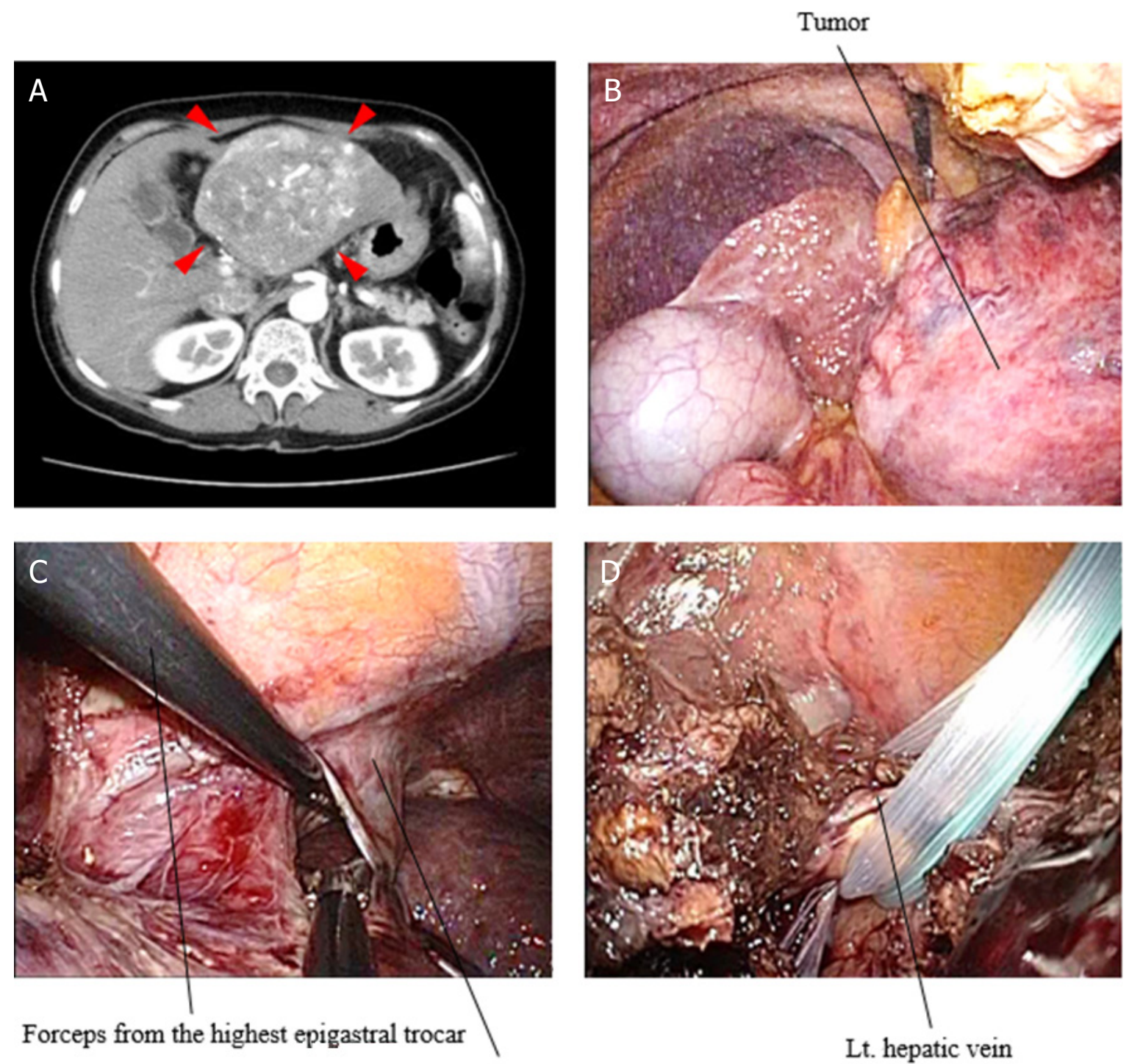

\section{Lt. triangular ligament}

Figure 10. Totally laparoscopic hemi-hepatectomy of left liver for large tumor. (A) A CT scan showed a hepatocellular carcinoma 12 $\mathrm{cm}$ in diameter, mainly located in left lateral section involving umbilical portion. There were several daughter nodules in segment 4; (B) due to the large tumor, left subphrenic space was not well visualized, but orientation of the hepatic hilar portion was preserved. After the individual isolation of the left and middle hepatic artery and the left portal vein, hepatic parenchyma was transected, prior to the mobilization of the left liver; (C) parenchymal transection allowed to tract the left liver to the caudal side; we put an additional trocar on the most cranial side of epigastrium. This was quite helpful for the division of the left triangle ligament; (D) the confluence of the left hepatic vein was also encircled by tape and divided using stapling device 


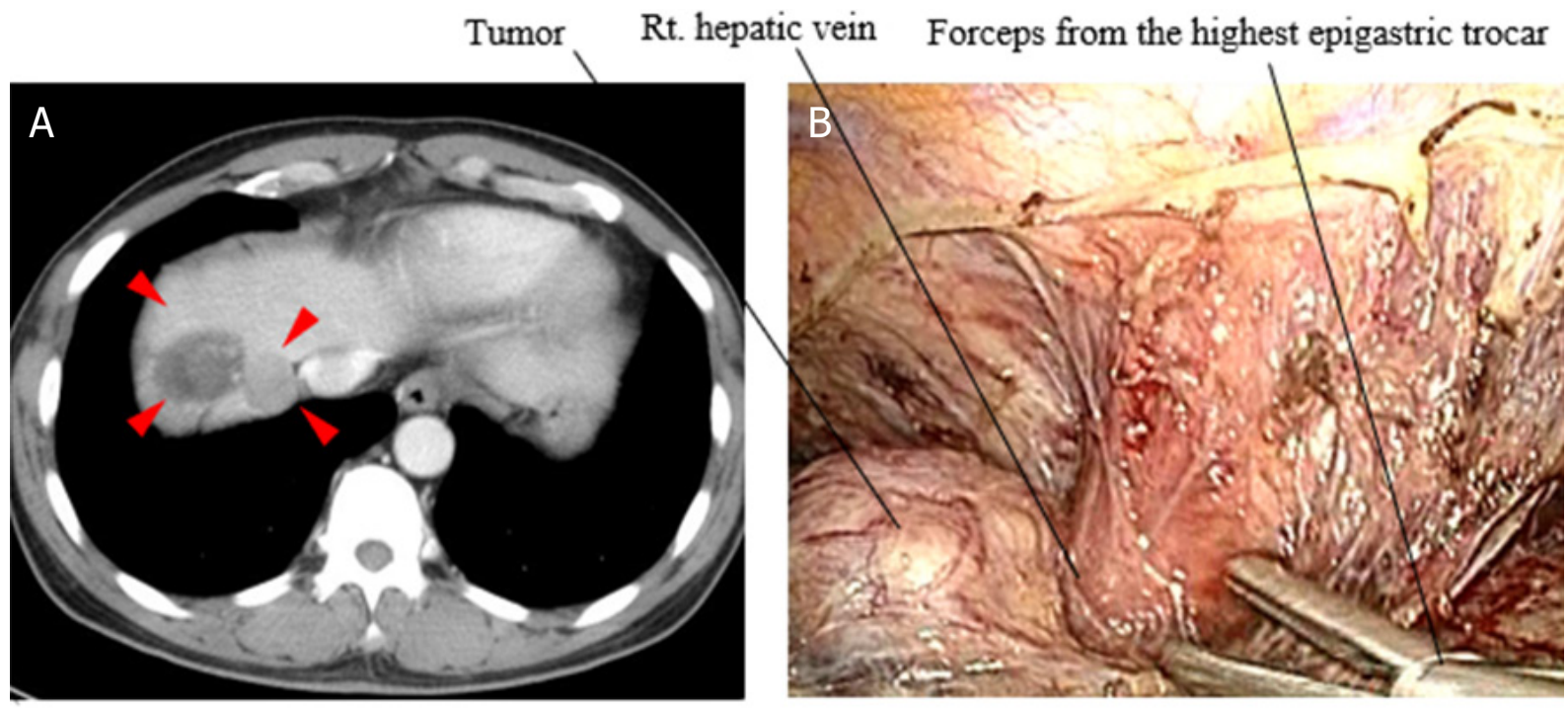

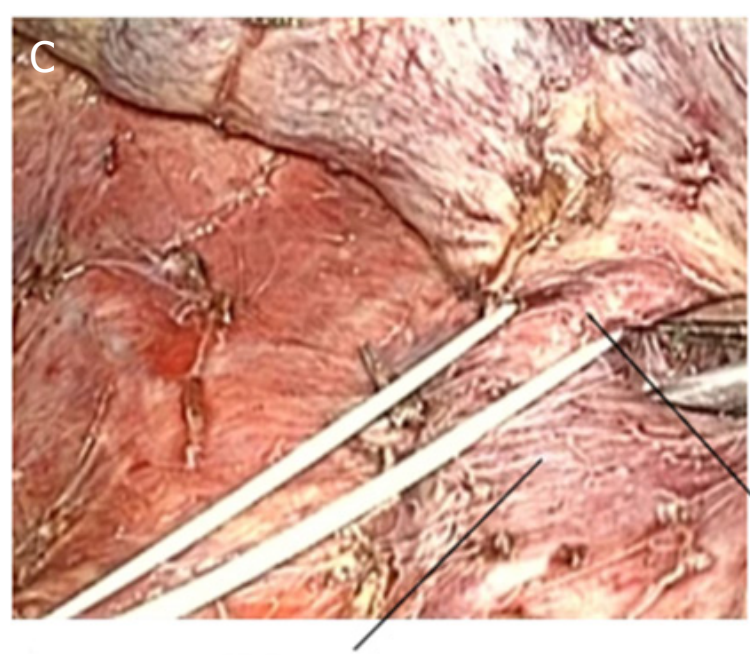

Inferior Vena Cava

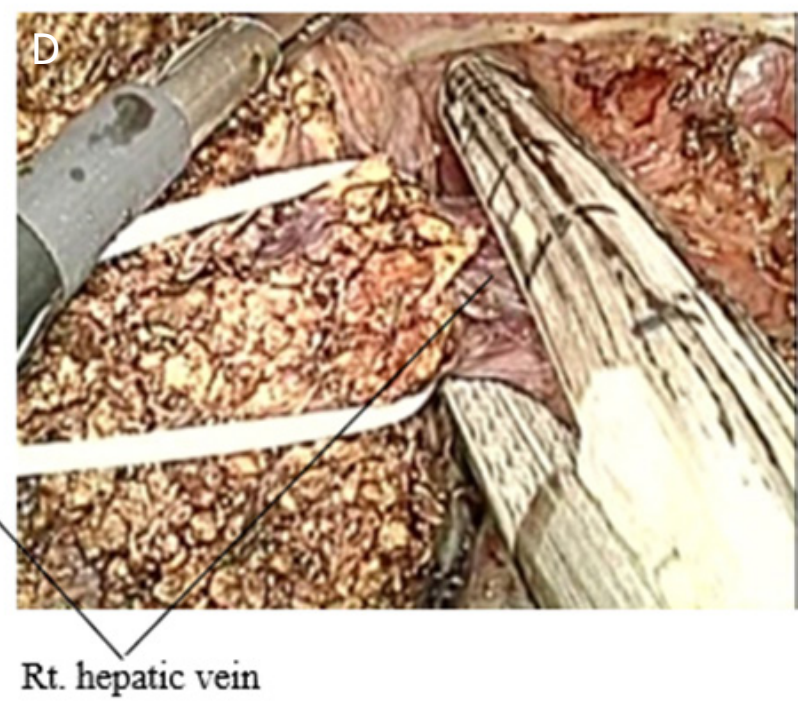

Figure 11. Totally laparoscopic hemi-hepatectomy of the right liver for hepatocellular carcinoma (HCC) located adjacent to the confluences of the right hepatic vein. (A) A CT scan showed multiple HCCs in the right liver. One of the tumors was located near the confluence of the right hepatic vein; (B) an additional trocar was placed at the highest portion of epigastrium to reach just to the tumor; (C) the right hepatic vein was successfully encircled extrahepatically; (D) hepatic parenchymal transection was performed by the usual fashion. The right hepatic vein was safely divided by tape retraction and stapling device

highest portion of epigastrium. Then, the confluence of the removed hepatic vein was carefully dissected by brunt dissection from both anterior and posterior side walls of the hepatic vein. If the confluence of the hepatic vein was skeletonized, vessel tape was encircled around the hepatic vein extrahepatically. Once the hepatic parenchymal transection was completed, confluence of the hepatic vein was sufficiently opened by retraction of vessel tape laterally. Then, stapling device was applied with appropriate distance from the tumor in all three cases [Figure 11A-D].

In two cases with suspicion of tumor invading the right diaphragm and the right adrenal gland, an additional trocar placement was also required to have favorable exposure and usage of energy device from the highest portion of epigastrium at the timing of mobilization of hemi-liver. Additionally, the flexible type laparoscope was an indispensable tool to obtain adequate exposure. When the dissection or removal of invaded tumor was insufficient, these procedures were performed at the last step of the hemi-hepatectomy [Figure 12A-D]. 

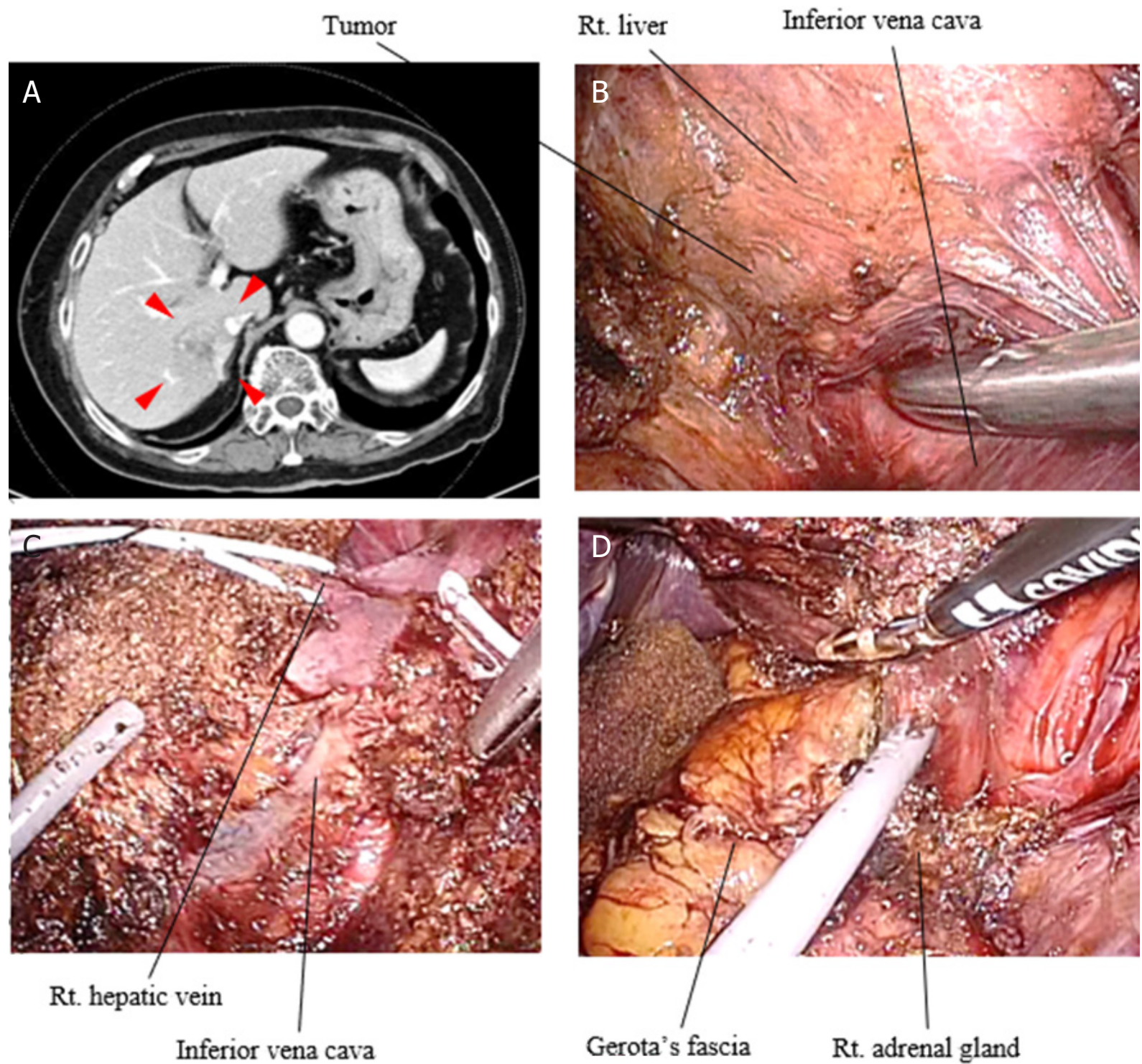

Figure 12. Totally laparoscopic hemi-hepatectomy of the right liver for cholangiocellular carcinoma located adjacent to the inferior vena cava with invasion to the right adrenal gland: (A) a CT scan showed a mass forming intrahepatic cholangiocarcinoma extended to just beside the inferior vena cava; (B) the tumor was also suspected of invasion to the right adrenal gland; (C) the right liver was transected prior to the complete mobilization; and (D) finally, the right liver was removed with partial right adrenalectomy from the retroperitoneum

In the case of suspicion of tumor invading to the retroperitoneum, conversion to hybrid technique with small laparotomy was required due to hemorrhage.

\section{DISCUSSION}

In our experience, the population of TLHHs for liver tumors including FIG and DIG was $4.3 \%$ of all liver resections, 9.4\% of all LLRs and $22.3 \%$ of all hemi-hepatectomies. Furthermore, FIG of TLHHs was limited to $11.7 \%$ of all 94 hemi-hepatectomies performed at our institution. Thus, favorable indication of TLHHs was stringently selected preoperatively.

Although several patients had only one tumor in our TLHH cases, their tumors required hemihepatectomy due to large size, located centrally in hemi-liver, nearby the first order branch of Glissonean pedicle, or growth with irregular margin. 
Laparoscopic caudal view with magnification can provide a better recognition of structures on hepatic hilum and dorsal area around the IVC than in the open surgery. Additionally, this approach can be suitable to maintain liver parenchymal transection plain made along the body axis, such as hemi-hepatectomies. Therefore, TLHHs can be suitable for technical standardization by laparoscopic procedure. However, many maneuvers are required to complete the procedures. TLHHs have been suggested as being able to be accomplished by surgical teams with extensive experiences in both open and laparoscopic liver surgery ${ }^{[10-12]}$. Therefore, the maintenance of good operative field with cooperation among the surgeon, assistant, and scopist is indispensable to standardize the procedures and overcome technical difficulties.

Five trocars with an additional extracorporeal access for Pringle's maneuver were usually placed in both left and right hemi-hepatectomies. As mentioned above, our standard formation of the surgeon and assistant standing on either side of the patient differs from others suggesting that the surgeon stands between the patient's legs ${ }^{[11]}$. Surgeon use two right-sided trocars of $5 \mathrm{~mm}$ and $12 \mathrm{~mm}$ in diameter, and the assistant uses trocars from epigastric and left lateral positions. We consider that our preferred formation can significantly contribute to stable teamwork with the first assistant.

One of the key points is a vascular division on hepatic hilum and major hepatic veins.

In the hilar dissection of TLHHs, we usually used individual vessels isolation technique, the so-called "control method", as a conventional intrafascial approach to separate the vessels in the hepato-duodenal ligament ${ }^{[13]}$. Although the extrafascial Glissonean pedicle approach ${ }^{[14]}$ has also been applied in the hilar dissection technique of $\mathrm{LH}^{[15]}$, we prefer the control method because of occasional instability in the laparoscopic division of Glissonean pedicle by using stapling device due to its thickness and limited angle, as well as the technical stability of the control method in our team. Here, vessel taping is extremely important for safe and precise division of the hilar vessels.

The tape retraction of the confluence of hepatic veins to the lateral side is a useful maneuver to apply the stapling device and divide large hepatic vein safely.

Our timing of liver mobilization differs from other authors, and several approaches to hepatocaval confluence are described ${ }^{[10,11,16]}$. When the tumor was not at risk of rupture and the liver parenchyma was soft, complete mobilization of the liver before parenchymal transection could be performed conventionally. If operative visualization was suitable, the confluence of right hepatic vein or common confluence of the middle and left hepatic vein could be encircled extrahepatically. When the isolation of bifurcation of large hepatic vein and the IVC was complicated, it could be postponed until after liver transection. Whenever these procedures were performed, the flexible angle laparoscope was an indispensable instrument. For a large tumor, with or without liver stiffness, anterior approach, which transected the liver prior to liver mobilization, could be required for the safe manipulation of the liver ${ }^{[16,17]}$.

We consider that there is no strict distinction in indication of TLHHs for tumor diameter; soft tumors larger than approximately $70 \mathrm{~mm}$ could be removed with conventional fashion, while hard tumors larger than $70 \mathrm{~mm}$ could be better transected by anterior approach. When the tumor was invading the retroperitoneum, right adrenal gland, or dorsal part of diaphragm, it could also be dissected close to the end of TLHH procedures, with consideration of requirement of hand-assisted or laparoscopy-assisted hybrid technique. However, the large tumor which firmly overlays the hepatic hilum is contraindication of TLHHs. In the retrieval of resected liver, it can be an issue regarding the prolonged operative duration, putting it into a plastic bag, and removing it from the limited length of skin incision.

Hepatic parenchymal resection should meticulously be performed by using a Cavitron ultrasonic surgical aspirator or a clamp crushing method under the guidance of intraoperative laparoscopic ultrasound. 
Liver transection technique based on the appropriate selection of devices is essential to achieve successful surgery $^{[18]}$.

Incidence of gas embolism in laparoscopic major liver resection has been reported to ranged $0.2 \%-1.5 \%{ }^{[19]}$. To avoid gas embolism in LH, therefore, we customarily use caution, maintaining intraperitoneal pressure around $10 \mathrm{mmHg}$, with fluctuation of the IVC in half-filled state ${ }^{[11]}$.

Intraoperative cholangiography prior to division of hepatic ducts is introduced to reveal anatomical variation or bile leakages. The recently developed Indocyanine green fluorescence imaging can be used in intraoperative cholangiography ${ }^{[20,21]}$.

The hand-assisted laparoscopic procedure and the laparoscopy-assisted hybrid procedure should remain options against unexpected trouble due to technical or oncological reason ${ }^{[22]}$. A conversion case to hybrid technique due to intraoperative hemorrhage in our series was safely coped with by prompt decision.

Although further improvement is necessary in our result of TLHHs in operative duration, blood loss, or postoperative morbidity, we believe TLHHs can be technically standardized. Additionally, technical modifications from stylized procedure, such as changes in the order of procedures, use of additional trocars for manipulation of surgical devices, or laparoscope, could contribute to performing TLHHs in difficult cases.

However, further accumulation of experience is necessary to improve the outcome of TLHHs and overcome the limitations.

We conclude that stringent patient selection for TLHHs is a notably important aspect, and, thereby, TLHHs can safely be stylized by totally laparoscopic fashion in daily practice. Ascertainment and correspondence for difficulty can be made through technical stylization of totally laparoscopic procedure in hemihepatectomies. However, there is an important limitation in this study due to the small number cases in each group, and further investigation is needed to clarify the feasibility of TLHHs in FIG and DIG.

\section{DECLARATIONS}

\section{Authors' contributions}

Conception and design of the study and performed data analysis and interpretation, drafting the article: Otsuka Y

Critical revise of the article: Kaneko $\mathrm{H}$

Read and approved the final version of the manuscript: Otsuka Y, Ito Y, Matsumoto Y, Kimura K, Kajiwara Y, Okubo K, Tsuchiya M, Okada R, Ishii J, Maeda T, Funahashi K, Kaneko H

\section{Availability of data and materials}

Not applicable.

\section{Financial support and sponsorship}

None.

\section{Conflicts of interest}

All authors declared that there are no conflicts of interest.

\section{Ethical approval and consent to participate}

Not applicable. 


\section{Consent for publication}

Not applicable.

\section{Copyright}

(c) The Author(s) 2021.

\section{REFERENCES}

1. Wakabayashi G, Cherqui D, Geller DA, et al. Recommendations for laparoscopic liver resection: a report from the second international consensus conference held in Morioka. Ann Surg 2015;261:619-29.

2. Takahara T, Wakabayashi G, Konno H, et al. Comparison of laparoscopic major hepatectomy with propensity score matched open cases from the National Clinical Database in Japan. J Hepatobiliary Pancreat Sci 2016;23:721-34.

3. Hwang DW, Han HS, Yoon YS, et al. Laparoscopic major liver resection in Korea: a multicenter study. J Hepatobiliary Pancreat Sci 2013;20:125-30.

4. Tzanis D, Shivathirthan N, Laurent A, et al. European experience of laparoscopic major hepatectomy. J Hepatobiliary Pancreat Sci 2013;20:120-4.

5. Dagher I, Gayet B, Tzanis D, et al. International experience for laparoscopic major liver resection. J Hepatobiliary Pancreat Sci 2014;21:732-6.

6. Kaneko H, Takagi S, Shiba T. Laparoscopic partial hepatectomy and left lateral segmentectomy: Technique and results of a clinical series Surgery 1996;120:468-75.

7. Otsuka Y, Tsuchiya M, Maeda T, et al. Laparoscopic hepatectomy for liver tumors: proposals for standardization. $J$ Hepatobiliary Pancreat Surg 2009;16:720-5.

8. Yamanaka N, Okamoto E, Oriyama T, et al. A prediction scoring system to select the surgical treatment of liver cancer. Further refinement based on 10 years of use. Ann Surg 1994;219:342-6.

9. Kanda Y. Investigation of the freely available easy-to-use software 'EZR' for medical statistics. Bone Marrow Transplant 2013;48:452-8.

10. O’Rourke N, Fielding G. Laparoscopic right hepatectomy: surgical technique. J Gastrointest Surg 2004;8:213-6.

11. Gayet B, Cavaliere D, Vibert E, et al. Totally laparoscopic right hepatectomy. Am J Surg 2007;194:685-9.

12. Han HS, Yoon YS, Cho JY, Ahn KS. Laparoscopic right hemihepatectomy for hepatocellular carcinoma. Ann Surg Oncol 2010;17:2090-1.

13. Honjo I, Araki C. Total resection of the right lobe of the liver; report of a successful case. J Int Coll Surg 1955;23:23-8.

14. Takasaki K. Glissonean pedicle transection method for hepatic resection: a new concept of liver segmentation. $J$ Hepatobiliary Pancreat Surg 1998;5:286-91.

15. Cho A, Yamamoto H, Kainuma O, et al. Extrahepatic Glissonean approach for laparoscopic major liver resection (with video). $J$ Hepatobiliary Pancreat Sci 2013;20:141-4.

16. Ratti F, Cipriani F, Catena M, Paganelli M, Aldrighetti L. Approach to hepatocaval confluence during laparoscopic right hepatectomy: three variations on a theme. Surg Endosc 2017;31:949.

17. Lai EC, Fan ST, Lo CM, Chu KM, Liu CL. Anterior approach for difficult major right hepatectomy. World J Surg 1996;20:314-7; discussion 318.

18. Otsuka Y, Kaneko H, Cleary SP, Buell JF, Cai X, Wakabayashi G. What is the best technique in parenchymal transection in laparoscopic liver resection? Comprehensive review for the clinical question on the 2nd International Consensus Conference on Laparoscopic Liver Resection. J Hepatobiliary Pancreat Sci 2015;22:363-70.

19. Otsuka Y, Katagiri T, Ishii J, et al. Gas embolism in laparoscopic hepatectomy: what is the optimal pneumoperitoneal pressure for laparoscopic major hepatectomy? J Hepatobiliary Pancreat Sci 2013;20:137-40.

20. Ishizawa T, Tamura S, Masuda $\mathrm{K}$, et al. Intraoperative fluorescent cholangiography using indocyanine green: a biliary road map for safe surgery. J Am Coll Surg 2009;208:e1-4.

21. Otsuka Y, Kaneko H. Usefulness of ICG fluorescence imaging in laparoscopic liver resection. ICG Fluorescence Imaging and Navigation Surgery. Tokyo: Splinger Japan; 2016. pp. 369-80.

22. Kaneko H, Otsuka Y, Kubota Y, Wakabayashi G. Evolution and revolution of laparoscopic liver resection in Japan. Ann Gastroenterol Surg 2017;1:33-43. 